\title{
Numerical Computation of Cone-Jet Formation and Deposition Characteristics in Electrostatic Spray
}

Wei Wei ${ }^{1}$, Sheng Wang $^{2 *}$, Zhaolin $\mathrm{Gu}^{1 *}$, Kiwamu Kase ${ }^{2}$, Yusuke Tajima ${ }^{2}$, Takaaki Orii ${ }^{2}$, Hideaki Takaku ${ }^{2}$, Noboru Imai ${ }^{2}$, Jungmyoung $\mathrm{Ju}^{2} \&$ Yutaka Yamagata ${ }^{2}$

1 Xi'an Jiaotong University, No.28, Xianning West Road, Xi'an, Shaanxi, 710049, China

2 RIKEN Institute, 2-1 Hirosawa, Wako City, Saitama 351-0198, Japan

*Corresponding-author E-mail: swang@riken.jp and guzhaoln@mail.xjtu.edu.cn

\begin{abstract}
Electrospray deposition (ESD) is a method to fabricate thin films of micro/nanoparticles directly from solution. It has been used in various processes including sample preparation in mass spectrometry, polymer coatings, organic film formation in solar cells, fuel cells and ink-jet printing. In this study a comprehensive ESD simulation methodology consisting of two solvers, i.e., Solver 1 for cone-jet formation and droplet generation modeling, and Solver 2 for droplet trajectory tracking and deposition characteristic calculations was developed. Without the assumption of the initial cone shape, the fluid flow equation and electric field distribution equation were solved simultaneously to obtain the evolution of cone-jet and droplets emitted from the cone-jet tip under different operation conditions. Based on the droplet characteristics from Solver 1, the trajectory and deposition characteristics of droplet/particle were investigated by using Solver 2 with a three dimensional Lagrangian model. In order to verify the validity of the proposed simulation methodology, ESD experiments were carried out. Various organic solvents were sprayed by using the ESD technique to investigate the effects of the applied voltage and the properties of the solvent to the deposition characteristics. The deposition area of the sprayed organic droplets was observed by using a CCD camera. The experimental data and the numerical results are consistent in the deposition characteristics. The method proposed in this study will be very helpful for fabricating thin films of micro/nanoparticles with ESD.
\end{abstract}

Keywords. Electrospray deposition, Cone-jet formation, Deposition characteristics, Numerical simulation

\section{INTRODUCTION}

Electrospray deposition (ESD) is a method to fabricate thin films of micro/nanoparticles directly from solution. It has attracted great attentions recently due to several advantages over the other deposition methods, such as high deposition efficiency, no ring stain effect during deposition process, high resolution and uniform patterning, the controllability of the size of dried nanoparticle and so on. It has been used in various processes including sample 
preparation in mass spectrometry[1], electric propulsion [2], food and pharmaceutical industries[3], electrostatic deposition[4], and et al.

ESD can be described as at least three different processes. The first process is the acceleration of the liquid in the liquid cone to form a cone-jet. This acceleration process and the shape of the liquid cone are the result of the force balance of surface tension, gravity, electric stresses in the liquid surface, inertia and viscous stresses. The second process is the breakup of the jet into droplets. The third process is the development of the spray after droplet production and the deposited on substrate.

To obtain an optimum deposition condition using electrospray device, many parameters have to be considered carefully, and a lot of efforts have to be made if it entirely relies on experiments $[5,6]$. Numerical simulation techniques have been developed greatly and become an indispensable tool in both academic field and industrial field [7, 8]. However, obtaining an optimum ESD processing condition still relies heavily on many trial experiments due to the complexity of the multi-physics phenomenon during the whole ESD process and the lack of a reliable numerical simulation technique. In this study, a comprehensive ESD simulation methodology covering the above three processes is developed. It mainly consists of two solvers: (1) solver for cone-jet formation and droplet generation, (2) solver for droplet trajectory tracking and deposition characteristics. In Solver (1) a coupling numerical scheme of Navier-Stokes system of the sample liquid and the Gauss theory for the electric field is adopted and used to calculate the jet velocity and the mother droplet's diameter, charge, position and velocity. The results from Solver (1) will serve as an initial condition of Solver (2) to obtain the droplet/particle trajectory and deposition characteristics. The two Solvers are combined as an organic whole. The deposition characteristics under different geometrical dimension of ESD device, different applied voltages, as well as different spraying materials are investigated. This study will be very helpful for fabricating thin films of micro/nanoparticles with ESD.

\section{SOLVER FOR CONE-JET FORMATION AND DROPLET GENERATION}

In electrohydrodynamics, inertial force, viscosity, and electric force are relevant to flow behavior. Conservation of mass and momentum for an incompressible flow are given as

$$
\begin{gathered}
\nabla \cdot \mathbf{U}=0 \\
\frac{\partial(\rho \mathbf{U})}{\partial t}+\nabla \cdot(\rho \mathbf{U U})=-\nabla p+\nabla \cdot\left(\sigma^{\mathrm{f}}+\sigma^{\mathrm{e}}\right)+\mathrm{f}_{b}
\end{gathered}
$$

where $\mathbf{U}$ is the velocity field, $p$ is the hydrodynamic pressure, $\rho$ is the mass density, $\mathrm{f}_{\mathrm{b}}$ are body forces. The viscous stress tensor, $\sigma^{\mathrm{f}}$, is given by

$$
\sigma^{\mathrm{f}}=\mu\left[\nabla \mathbf{U}+(\nabla \mathbf{U})^{T}\right]-\frac{2}{3} \mu(\nabla \cdot \mathbf{U}) \mathbf{I}
$$

where $\mathbf{I}$ is the identity tensor and $\mu$ is the dynamic viscosity. The Maxwell stress tensor, $\sigma^{\mathrm{e}}$, and the corresponding electric body force, $\mathrm{f}^{\mathrm{e}}$, are described by 


$$
\begin{gathered}
\sigma^{e}=\varepsilon \varepsilon_{0} \mathbf{E} \mathbf{E}-\frac{\varepsilon \varepsilon_{0}}{2} \mathbf{E} \cdot \mathbf{E}\left(1-\frac{\rho}{\varepsilon \varepsilon_{0}} \frac{\partial \varepsilon \varepsilon_{0}}{\partial \rho}\right) \mathbf{I} \\
\mathrm{f}_{\mathrm{e}}=\nabla \cdot \sigma^{\mathrm{e}}=q_{\mathrm{V}} \mathbf{E}-\frac{1}{2} \mathbf{E}^{2} \nabla \varepsilon \varepsilon_{0}+\nabla\left(\frac{1}{2} \rho \frac{\partial \varepsilon \varepsilon_{0}}{\partial \rho} \mathbf{E}^{2}\right)
\end{gathered}
$$

where $\varepsilon$ is the electric permittivity and $\varepsilon_{0}=8.85 \times 10^{-12} \mathrm{C} / \mathrm{Vm}$ is the permittivity of the vacuum, $q_{\mathrm{V}}$ is a volumetric electric charge density, and $\mathbf{E}$ is the electric field vector.

The first term on the right-hand of Eq.(5) is the polarization stress acting along the normal direction of the interface as a result of the term $\nabla \varepsilon$. The second term describes the interaction of the electric charges with the electric field, acting along the direction of the electric field. The last term results from the changes in material density, usually the so-called electrorestriction force density. This term is neglected in this study as the fluid is assumed to be incompressible.

In electrohydrodynamics, dynamic currents are small and therefore the magnetic induction effects can be ignored. Thus, the electric field intensity $\mathbf{E}$ is irrotational $(\nabla \times \mathbf{E}=0)$. The Gauss law in a dielectric material of relative permittivity $\varepsilon$ can be written in terms of the electric displacement $\left(\mathbf{D}=\varepsilon \varepsilon_{0} \mathbf{E}\right)$ as,

$$
\nabla \cdot \mathbf{D}=q_{\mathrm{V}}
$$

The electric field intensity $\mathbf{E}$ can be expressed as the gradient of the scalar electric potential, $\phi$, as

$$
\mathbf{E}=-\nabla \phi
$$

The charge conservation equation is given by

$$
\frac{D q_{V}}{D t}+\nabla \cdot(K \mathbf{E})=0
$$

where $K$ is the electrical conductivity of the medium. $D / D t=\partial / \partial t+\mathbf{U} \cdot \nabla$ is the material derivative.

The tangential component of the electric field intensity is continuous across the interface, that is $\mathbf{n} \times\|\mathbf{E}\|=0$, where $\|\cdot\|$ represents a jump (medium-1 - medium-2) across the interface and $\mathbf{n}$ is the unit normal vector at the interface. The normal component of the electric displacement vector is discontinuous at the interface and the jump is given by,

$$
\mathbf{n} \cdot\|\mathbf{D}\|=q_{\mathrm{s}}
$$

where $q_{\mathrm{s}}$ is the free charge per unit surface area.

In the conventional volume-of-fluid (VOF) method[9], the transport equation for an indicator function, representing the volume fraction of one phase, is solved simultaneously with the continuity and momentum equations,

$$
\frac{\partial \gamma}{\partial t}+\nabla \cdot(\mathbf{U} \gamma)=0
$$

where $\gamma$ is the phase fraction. The phase fraction $\gamma$ can take values within the range $0 \leq \gamma$ $\leq 1$, with the values of zero and one corresponding to regions accommodating only one phase, 
e.g., $\gamma=0$ for gas and $\gamma=1$ for liquid. Accordingly, gradients of the phase fraction are encountered only in the region of the interface.

The physical properties are calculated as weighted averages based on the distribution of the liquid volume fraction, thus being equal to the properties of each fluid in their corresponding occupied regions and varying only across the interface,

$$
\begin{gathered}
\rho=\rho_{l} \gamma+\rho_{g}(1-\gamma) \\
\mu=\mu_{l} \gamma+\mu_{g}(1-\gamma) \\
K=K_{l} \gamma+K_{g}(1-\gamma) \\
\varepsilon=\varepsilon_{l} \gamma+\varepsilon_{g}(1-\gamma)
\end{gathered}
$$

where $l$ and $g$ are densities of liquid and gas, respectively.

For a leaky-dielectric fluid [10] the charge conservation equation in the bulk of an inhomogeneous continuous media Eq.(8) can be assumed to attain steady state in a time much smaller than the time scale of the fluid motion $\left(\tau_{P} ? \tau_{C}\right)$ and can thus be simplified as

$$
\nabla \cdot(K \mathbf{E})=0
$$

The stress induced in the medium due to applied electric field is given by Maxwell stress tensor (Eq.(4)) and the equivalent volume force representation is given by

$$
\mathrm{f}_{\mathrm{e}}^{\mathrm{V}}=q_{\mathrm{V}} \mathbf{E}-\frac{1}{2} \mathbf{E}^{2} \nabla \varepsilon \varepsilon_{0}
$$

where $q_{\mathrm{V}}=\nabla \cdot\left(\varepsilon \varepsilon_{0} \mathbf{E}\right)$ is the volume charge density.

The boundary conditions at the interface are continuity of the potential $(\|\phi\|=0)$.

The charge transfer on the cone-jet is induced by two ways, i.e., conduction and surface charge convection. On the cone base, the charges are mainly transferred through conduction, while on the jet the charges are moved by convection. Therefore, the total current $(I)$ generated by the cone-jet can be described by Eq.(17), as[11] :

$$
I=\int\left(\pi R^{2}(z) K E_{s}(z)+2 \pi R(z) u(z) q_{s}\right) d z
$$

where $R(z)$ is the cone-jet radius, $E_{s}(z)$ is the electrical field intensity, $u(z)$ is the velocity and $q_{\mathrm{s}}$ is the charge density on the cone-jet surface.

As regards the present problem, the flow field and electric fields are tightly coupled through complex interactions. The fluid motions are influenced by electrical field and surface charge density. In turn, these motions modify the charge density distribution along the interface through the convective term of the current density in the corresponding charge conservation equation. The electric potential fields are determined by the surface charge distributions, and the motion may substantially change the value of the electric fields.

The electric field is first solved using Eq.(15) (except for the air-liquid interface) and the charge distribution along the surface is determined by Eq.(9). The flow field Eqs.(1), (2)and 
(10) are solved numerically using a finite volume method to get the velocity field and to track the interface of liquid-gas until a stable cone-jet is formed.

(a)

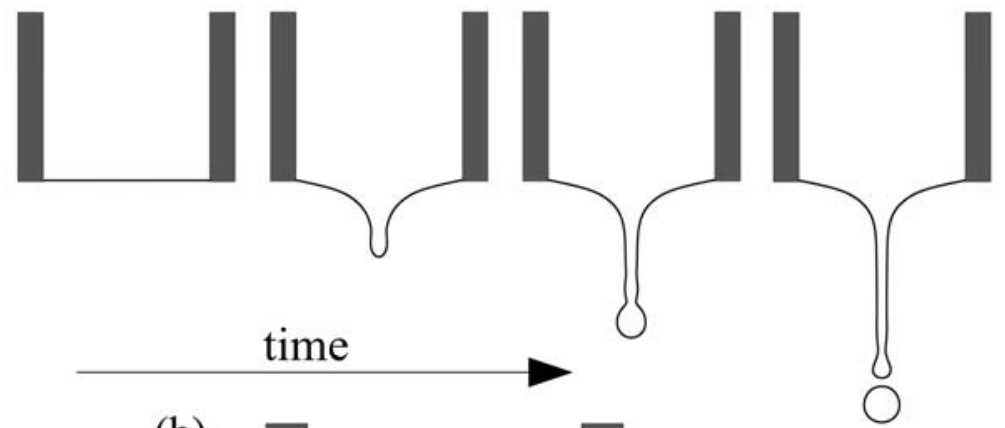

(b)

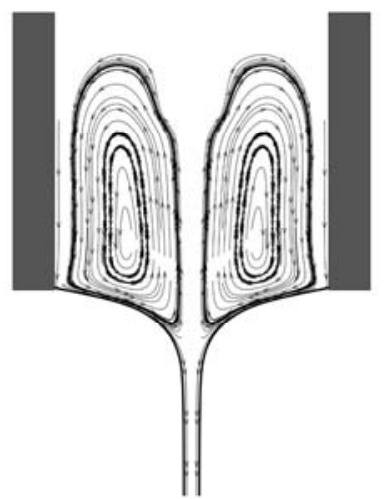

Fig. 1 Formation of cone-jet. (a) Evolution of cone-jet; (b) Velocity streamline in cone.

Based on the physical properties of the liquid, such as density $\rho$, viscosity $\mu$, conductivity $K$, dielectric constant $\varepsilon$ and the surface tension coefficient $\sigma$, flow rate $Q$, and the electrode configuration, the formation of cone-jet and generation of droplet are obtained, as shown in Fig. 1. The droplet characteristics, such as droplet's diameter, charge, position and velocity, are also calculated with this Solver.

Under the effect of the external electric field, gravity and surface tension, two symmetrical circulations inside the capillary are generated as shown in Fig. 1(b). The electric field within the liquid repels the free charges to the surface of the cone, and thus the surface is charged. In addition, subjected to the normal electric field, the liquid interface has a shrink. Simultaneously the fluid moves along the interface at tangential direction. Then a jet with diameter smaller than the capillary diameter is formed as shown in Fig. 1. Due to the surface tension, the interface at the end of the jet shrinks and an unstable wave-like structure with several "necks" appears. With the continuous effect of the electric force, the "neck" jet flows downward with high speed, then the liquid interface becomes thinner and thinner, and finally breaks into droplets.

\section{SOLVER FOR DROPLET/PARTICLE TRAJECTORY AND DEPOSITION CHARACTERISTICS}

A three-dimensional Lagrangian model based on the works of [12] was used in this study under the assumption of spherical particles. Brownian motion of particles can be negligible, because the particle kinetic energy caused by the electric field is much greater than random thermal energy [13]. The equation for the motion of the particle $i$ based on Newton's second 
law can be written as follows:

$$
\frac{\pi}{6} d_{i}^{3} \rho_{d} \frac{d v}{d t}=C_{D} \frac{\pi}{8} \rho_{g} d_{i}^{2}\left|v_{i}^{\mathbf{v}}\right|^{2}+q_{i} \mathbf{E}+\frac{1}{4 \pi \varepsilon_{0}} \sum_{i, j, i \neq j} \frac{q_{i} q_{j}}{\left|r_{i j}\right|^{\mathbf{v}}} r_{i j}+\frac{\pi}{6} d_{i}^{3} \rho_{d} \underset{\mathbf{w}}{r_{1}}
$$

The left-hand side of Eq. (18)accounts for the inertia of the particle $i$. The terms on the right-hand side account for the drag force by the surrounding gas, the electrical forces exerted by the external electric field, the mutual electrical repulsion forces between the charged particles, and the gravitational force, respectively.

The drag coefficient of Newton's resistance law can be expressed as follows:

$$
C_{D}=\frac{24}{R e}\left(1+0.15 R e^{0.678}\right) \quad \text { for } R e<800
$$

where $R e$ is the Reynolds number.

In electrohydrodynamics, the dynamic currents are small, and hence the magnetic induction effects can be ignored. For simplicity, the effect of charged droplets self-induced electric field to external electric field is omitted[14], so the electric field in this study is given as Eq.(7).

In the present study, the particles with the diameters of Gaussian distribution are assumed to be electrically charged even before entering the electric field. The droplet diameter can be calculated in the Solver (1). As a highly charged droplet evaporates, its mass decreases while the number of charges on the droplet remains constant; thus, the surface charge density increases. Simultaneously, the electrostatic repulsion between charges with a same polarity increases at the droplet surface.

Above a critical point, known as the Rayleigh limit as expressed by Eq. (20) [15], the droplet will become unstable and will release charge through a series of fissions. Therefore, value of charge for each droplet must be smaller than the Rayleigh limit.

$$
q_{R}=\left(8 \pi^{2} \varepsilon_{0} \gamma d_{i}^{3}\right)^{1 / 2}
$$

The average charge per droplet was calculated using the spray current $I$ and liquid flow rate $Q$ by:

$$
q_{\text {ave }}=\frac{\pi}{6} d_{\text {ave }}^{3} \cdot \frac{I}{Q}
$$

where $q_{\text {ave }}$ is the average charge per droplet, $d_{\text {ave }}$ is the average droplet diameter and $I$ can be determined by Eq.(17). The charge on the randomly generated droplets was calculated using the relationship between the droplet size and the droplet charge [12]:

$$
\frac{q_{i}}{q_{\text {ave }}}=\left(\frac{d_{i}}{d_{\text {ave }}}\right)^{3}
$$

All the droplets/particles are generated near the tip of capillary and randomly distributed in $x$ and $y$ directions with the initial speed of $(0,0, w)$. Because of the same charge polarity, there is no interaction like collision or coagulation/agglomeration between particles. 


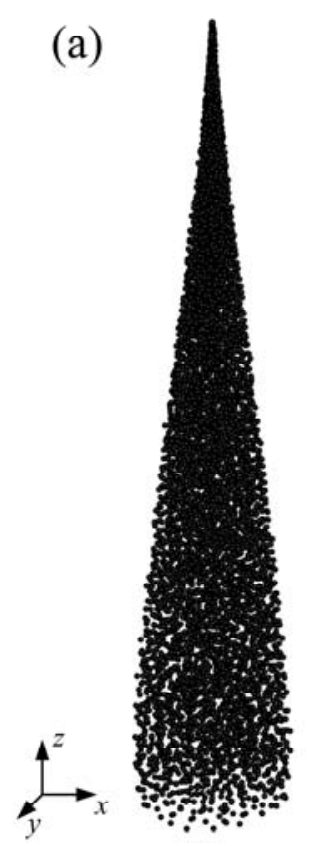

(b)

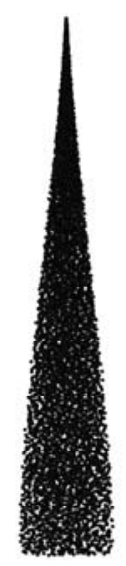

(c)

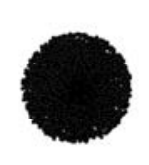

Fig. 2 Droplet/particle trajectory and deposition characteristic. (a) Isometric view. (b) Front view. (c) Top view.

Fig. 2 shows the droplet/particle trajectories and deposition characteristics using Solver (2). Each point represents an emitted droplet. The spray shape is a cone and the deposition shape on the substrate is a circular. The simulation results are consistent with the experimental results[16].

\section{CONCLUSION}

In this study, a comprehensive ESD simulation methodology covering the whole ESD process is developed. Without the assumption of the initial cone shape, the fluid flow equation and electric field distribution equation are solved simultaneously to obtain the evolution of cone-jet and droplets emitted from the cone-jet tip at various applied voltage and flow rate operation conditions. The droplet characteristics, such as droplet's diameter, charge, position and velocity, are calculated. Then the trajectory and deposition characteristics of droplet/particle were investigated by using a three-dimensional Lagrangian model. The spray shape and deposition shape is consistent with the experimental result. The method proposed in this study will be very helpful for fabricating thin films of micro/nanoparticles with ESD.

\section{Acknowledgment}

This work was funded by Grant-in-Aid for Young Scientists (No. 23760070) sponsored by Japan Society for the Promotion of Science.

\section{REFERENCES}


[1] Fenn J., et al., "Electrospray ionization for mass spectrometry of large biomolecules". Science.246, 64-71, 1989.

[2] Guerrero I., et al., "Ion evaporation from Taylor cones of propylene carbonate mixed with ionic liquids". J. Fluid. Mech.591, 437-459, 2007.

[3] Rezvanpour A. and C.-H. Wang, "Computational and experimental studies of electrospray deposition process in pharmaceutical micro-pattern formation". Chem. Eng. Sci.66, 3836-3849, 2011.

[4] Ju J., Y. Yamagata, and T. Higuchi, "Thin-Film Fabrication Method for Organic LightEmitting Diodes Using Electrospray Deposition". Adv. Mater.21, 4343-4347, 2009.

[5] Kim J. H., et al., "Direct pattern formation of bacterial cells using micro-droplets generated by electrohydrodynamic forces". Microfluidics and Nanofluidics.7, 829-839, 2009.

[6] Barletta M. and A. Gisario, "Electrostatic spray painting of carbon fibre-reinforced epoxy composites". Prog. Org. Coat.64, 339-349, 2009.

[7] Oh H., K. Kim, and S. Kim, "Characterization of deposition patterns produced by twinnozzle electrospray". J. Aerosol. Sci.39, 801-813, 2008.

[8] Wilhelm O., L. Madler, and S. E. Pratsinis, "Electrospray evaporation and deposition". J. Aerosol. Sci.34, 815-836, 2003.

[9] Hirt C. W. and B. D. Nichols, "Volume of fluid(VOF) method for the dynamics of free boundaries". J. Comput. Phys.39, 201-225, 1981.

[10] Saville D. A., "Electrohydrodynamics: The Taylor-Melcher leaky dielectric model". Annu. Rev. Fluid. Mech.29, 27-64, 1997.

[11] GananCalvo A. M., "Cone-jet analytical extension of Taylor's electrostatic solution and the asymptotic universal scaling laws in electrospraying". Phys. Rev. Lett.79, 217-220, 1997.

[12] Ganan-calvo A. M., et al., "The electrostatic spray emitted from an electrified conical meniscus". J. Aerosol. Sci.25, 1121-\&, 1994.

[13] Kim H., et al., "Parallel patterning of nanoparticles via electrodynamic focusing of charged aerosols". Nat Nano.1, 117-121, 2006.

[14] Hartman R. P. A., et al., "Electrohydrodynamic atomization in the cone-jet mode physical modeling of the liquid cone and jet". J. Aerosol. Sci.30, 823-849, 1999.

[15] Gomez A. and K. Q. Tang, "Charge and fission of droplets in electrostatic sprays". Phys. Fluids. 6, 404-414, 1994.

[16] Tang K. and A. Gomez, "On the structure of an electrostatic spray of monodisperse droplets". Phys. Fluids. 6, 2317-2332, 1994. 